\title{
Adoção de Realidade Aumentada no Ensino de Resistência dos Materiais
}

\author{
João Carlos Sedraz Silva ${ }^{1}$, Fernando da Fonseca de Souza ${ }^{2}$, \\ Luciano Sedraz Silva ${ }^{3}$, Jorge Luis Cavalcanti Ramos ${ }^{1}$. \\ Universidade Federal do Vale do São Francisco (Univasf) ${ }^{1}$ \\ Universidade Federal de Pernambuco (UFPE) ${ }^{2}$ \\ Universidade Federal de Sergipe (UFS) ${ }^{3}$ \\ joao.sedraz@univasf.edu.br, fdfdecin.ufpe.br \\ sedraz@bol.com.br, jorge.cavalcanti@univasf.edu.br
}

\begin{abstract}
This work aims to analyze the adoption of Augmented Reality - AR in teaching Engineering, from the students perspective of Mechanics of Materials discipline offered by the Universidade Federal do Vale do São Francisco, with the guiding element the Technology Acceptance Model - TAM. The research involved the participation of 50 students who, initially, conducted an experiment following a script for AR features tests, associated with the content of the discipline, and then answered a questionnaire based on TAM. The results indicate that the resources were well received by the students and that the adoption of AR contributes to the teaching of Mechanics of Materials, helping the presentation and understanding of the content.
\end{abstract}

Resumo. Este trabalho tem como objetivo analisar a adoção de Realidade Aumentada - RA no ensino de Engenharia, a partir da perspectiva de estudantes da disciplina Resistência dos Materiais ofertada pela Universidade Federal do Vale do São Francisco, tendo como elemento norteador o Modelo de Aceitação de Tecnologia - TAM. A pesquisa contou com a participação de 50 discentes que, inicialmente, realizaram um experimento seguindo um roteiro para testes de recursos de $R A$, associados ao conteúdo da disciplina, e, em seguida, responderam a um questionário fundamentado no TAM. Os resultados indicam que os recursos tiveram uma boa aceitação e que a adoção de $R A$ contribui para o ensino de Resistência dos Materiais, ajudando na apresentação e compreensão do conteúdo.

\section{Introdução}

O ensino de Engenharia, em muitas disciplinas, apresenta-se como um grande desafio para professores e estudantes, devido ao elevado nível de abstração envolvido nos conceitos abordados nesses cursos. Especificamente, na disciplina de Resistência dos Materiais, algumas alternativas para enfrentar esse desafio já foram propostas, em especial o uso de modelos qualitativos [Santos, 1983] e de ambientes virtuais de aprendizagem [Silva, 2013]

Os modelos qualitativos são protótipos físicos que permitem visualizar o comportamento de elementos estruturais sujeitos a um carregamento e, geralmente, são utilizados apenas em sala de aula, para complementar os cálculos teóricos. Os ambientes virtuais de aprendizagem funcionam como interface de comunicação e repositório de objetos de aprendizagem (simuladores, vídeos e textos complementares), que são 


\section{CBIE-LACLO 2015}

Anais dos Workshops do IV Congresso Brasileiro de Informática na Educação (CBIE 2015)

utilizados pelos estudantes, principalmente, fora do contexto das aulas/encontros presenciais [Dantas et al. 2014].

Uma outra alternativa para potencializar o ensino de Engenharia é a adoção de Realidade Aumentada - RA, que vem sendo estudada por vários pesquisadores e oferece novas aplicações em diversas áreas, como navegação, entretenimento, comércio, medicina, militar e educação.

$\mathrm{Na}$ educação, a RA fornece a professores e estudantes um método intuitivo e colaborativo de aprender, com novos canais e oportunidades para a geração de conhecimento, em qualquer contexto, dentro ou fora de sala de aula.

Segundo Valente (2005), para se utilizar as novas tecnologias da informação e da comunicação no ensino, são necessários, além dos dispositivos tecnológicos, dos softwares e da internet, professores com domínio pedagógico e capazes de refletir sobre as formas adequadas de utilização dos recursos como meio educacional.

É importante ressaltar que a utilização de novas tecnologias requer uma mudança de comportamento não apenas dos discentes, mas, principalmente, dos docentes, que serão condutores do processo de mudança. Para Haguenauer (2012), a implantação e utilização de aparatos tecnológicos devem ser precedidas e acompanhadas de estudos, para o desenvolvimento de novas metodologias, com foco na melhoria do processo de ensino-aprendizagem.

Pretende-se, com este trabalho, contribuir com o conjunto de estudos relacionados na área de Realidade Aumentada e suas possibilidades pedagógicas, com vistas ao desenvolvimento de novas metodologias de ensino-aprendizagem na Engenharia.

Portanto, o objetivo geral deste trabalho é analisar a adoção de Realidade Aumentada no ensino de Engenharia. Para tanto, utilizando como elemento norteador o Modelo de Aceitação de Tecnologia [Davis, 1989], o estudo é focado na perspectiva dos estudantes da disciplina Resistência dos Materiais, ofertada aos cursos de Engenharia da Universidade Federal do Vale do São Francisco - Univasf.

A escolha da disciplina deve-se a sua presença nos currículos da ampla maioria dos cursos de Engenharia das universidades brasileiras, e também a experiência de um dos autores deste trabalho como docente de Resistência dos Materiais.

Sendo assim, além desta introdução, o trabalho está organizado com mais cinco seções, que apresentam a fundamentação deste artigo, o método utilizado, a discussão dos resultados e as considerações finais da pesquisa.

\section{Realidade Aumentada}

Segundo Kirner (2007), a Realidade Aumentada - RA é a inserção de objetos virtuais no ambiente físico, mostrados ao usuário, em tempo real, com o apoio de algum dispositivo tecnológico, usando a interface do ambiente real, adaptada para visualizar e manipular os objetos reais e virtuais.

Para Milgram (1994), RA é uma parte da Realidade Mista (Figura 1), que representa um contínuo limitado pelo "ambiente real" e o "ambiente virtual". Entre esses extremos, inclui-se a Realidade Aumentada, em que objetos virtuais são 
visualizados em um ambiente circundante real, e Virtualidade Aumentada, em que as imagens de objetos reais são inseridas no mundo, predominantemente, virtual.

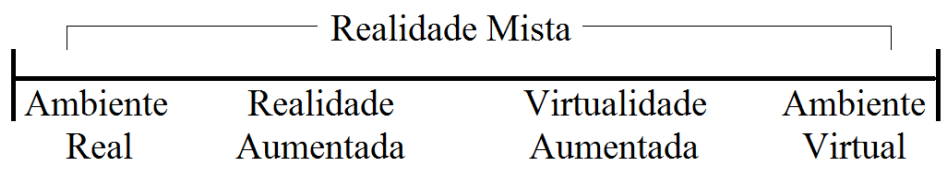

Figure 1. Realidade Mista.

\subsection{Questões de Percepção}

De forma mais estrita, a realidade não pode mudar/aumentar, mas sim a percepção do usuário [Normand, 2012]. Nesse contexto, a percepção é compreendida como a forma que o usuário reconhece e interpreta uma cena do ambiente no qual está inserido.

De acordo com Kruijff (2010), algumas questões de percepção devem ser destacadas, do ambiente real a ser capturado até a sobreposição dos gráficos visualizados pelo usuário.

- Questões do Ambiente: estrutura, cores e iluminação, são aspectos ambientais que podem causar problemas na visualização de imagens em dispositivos portáteis;

- Questões de Captura: resolução, campo de visão, distância focal, tempo de exposição e outros fatores relacionados à digitalização do ambiente em vídeo podem prejudicar a compreensão do mundo real;

- Questões do Aumento: erros de registro, oclusão e diferença de renderização dificultam a interpretação correta da cena;

- Questões dos Dispositivos de Exibição: campo de visão, brilho, contraste, cores, resolução, latência e reflexões, são problemas técnicos recorrentes nos displays;

- Questões dos Usuários: diferenças individuais que causam distorções de profundidade e visões distintas.

No uso de recursos de RA em dispositivos portáteis, como celulares e tablets (dispositivos utilizados nesta pesquisa), essas questões devem ser avaliadas e, quando necessário, devem ser promovidos ajustes para que a percepção dos usuários não seja prejudicada, o que pode anular a utilidade dos recursos.

\subsection{Interfaces Naturais de Usuário}

As Interfaces Naturais de Usuário (NUI) representam a evolução das interfaces gráficas, capazes de reduzir o tempo de aprendizado e melhorar a usabilidade dos sistemas. $\mathrm{O}$ ganho na curva de aprendizagem dessas interfaces está relacionado ao foco de seus projetados, que são centrados na reutilização de habilidades dos usuários para permitir a interação direta com o conteúdo. Como exemplos de NUIs amplamente difundidas nos dias atuais, podemos citar as telas sensíveis ao toque em tablets [Kawamoto, 2013].

Para Blake (2013), o projeto de NUIs devem considerar as seguintes diretrizes:

- Experiência instantânea: aproveitar habilidades específicas do domínio e habilidades comuns a todos os homens; 


\section{CBIE-LACLO 2015}

Anais dos Workshops do IV Congresso Brasileiro de Informática na Educação (CBIE 2015)

- Carga Cognitiva: reutilizar habilidades simples, já existentes, como aplicações que utilizam comando por toque ou apontadores semelhantes ao mouse;

- Aprendizagem progressiva: prever uma curva de aprendizagem suave, a partir de tarefas básicas até as tarefas avançadas;

- Interação direta: manipular diretamente o conteúdo dentro do contexto, evitando processos que envolvem inúmeros passos para a utilização de um recurso.

A partir das diretrizes apresentadas, o desenvolvimento de aplicações de RA terá maior chance de sucesso, podendo alcançar níveis superiores de aceitação nos grupos de usuários da solução. Uma estratégia para avaliar a aceitação de recursos de RA é o Modelo de Aceitação de Tecnologia - TAM, descrito na seção a seguir.

\section{Modelo de Aceitação de Tecnologia}

O Modelo de Aceitação de Tecnologia (Technology Acceptance Model) foi projetado para avaliar as causas da aceitação de sistemas computacionais, a partir da percepção dos usuários em relação à utilidade e à facilidade de uso das soluções [Davis, 1989].

Os construtos que fundamentam o TAM estão apresentados na Figura 2. O núcleo do modelo é constituído por dois importantes indicadores de aceitação: a facilidade de uso percebida e a utilidade percebida, que, respectivamente, referem-se ao "grau em que os usuários acreditam que a adoção de uma determinada tecnologia seria livre de esforço" e ao "grau em que uma pessoa acredita que a utilização de um determinado sistema melhoraria o seu desempenho no trabalho" [Persico et al., 2014].

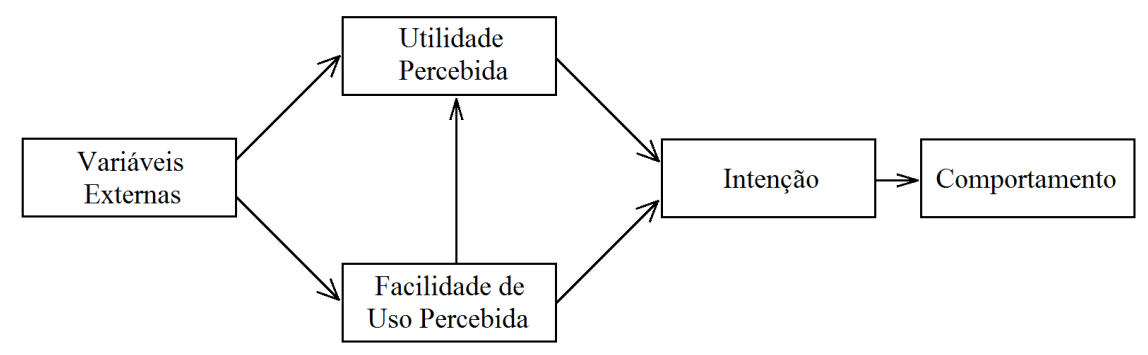

Figure 2. Modelo de Aceitação de Tecnologia.

A intenção de uso é o fator determinante para o sucesso da adoção dos sistemas de informação [Dias, 2011]. Essa intenção está relacionada com a utilidade percebida e a facilidade de uso percebida, cada uma exercendo um peso relativo. Além disso, é importante considerar as variáveis externas, que podem influenciar o contexto específico de uso da tecnologia [Ayeh et al., 2013].

O modelo oferece bases para elaboração de questões, que identificam indícios da percepção dos usuários, em relação ao uso de um determinado sistema. Nesse sentido, trabalhos recentes relacionam a aplicação do TAM na condução de pesquisas sobre a adoção de recursos de Realidade Aumentada [Haugstvedt e Krogstie, 2012], [Wojciechowski e Cellary, 2013].

\section{Método}

\subsection{Contexto do Estudo}

Resistência dos Materiais é uma disciplina prevista nas diretrizes dos cursos de Engenharia, que na Univasf está no conjunto de componentes curriculares obrigatórias. 
O objetivo geral dessa disciplina é capacitar o estudante para a avaliação e a resolução de problemas relacionados à análise de estruturas mecânicas, sujeitas a diferentes tipos de solicitações.

Conforme o Programa de Unidade Didática, na Univasf, a disciplina Resistência dos Materiais é planejada com uma carga-horária de sessenta horas, distribuída em três etapas. A cada etapa, por meio do ambiente virtual de aprendizagem, os estudantes têm acesso aos objetivos, o material de apoio (notas de aula, vídeo relacionado ao conteúdo ministrado em sala de aula e provas anteriores), necessário para acompanhamento das aulas presenciais e realização das atividades extraclasse.

\subsection{Objetivo}

Avaliar a aceitação dos estudantes em relação a adoção de recursos de Realidade Aumentada nas notas de aula da disciplina Resistência dos Materiais, ofertada na Univasf.

\subsection{Participantes}

No semestre em que a pesquisa foi realizada, a disciplina Resistência dos Materiais contou com noventa e quatro estudantes. Dentre esses, apenas 50 (cinquenta) estudantes responderam ao questionário aplicado na pesquisa, conforme indicado no Quadro 1.

\section{Quadro 1. Número de participantes por curso.}

\begin{tabular}{l|c}
\multicolumn{1}{c|}{ Curso } & Número de Estudantes \\
\hline Engenharia Agrícola e Ambiental & 06 \\
\hline Engenharia Civil & 12 \\
\hline Engenharia de Produção & 12 \\
\hline Engenharia Elétrica & 15 \\
\hline Engenharia Mecânica & 05 \\
\hline \multicolumn{1}{c}{ Total } & 50 \\
\hline
\end{tabular}

\subsection{Procedimentos}

Para atender o objetivo da pesquisa, inicialmente, foi realizado um experimento com os participantes, que seguiram um roteiro previamente definido para testes de três recursos de RA, associados a algumas notas de aula já ministradas em Resistência dos Materiais (Figura 3). Posteriormente, foi aplicado um questionário (Quadro 2), com os mesmos participantes, a fim de obter suas opiniões acerca da adoção de recursos de RA na disciplina Resistência dos Materiais.

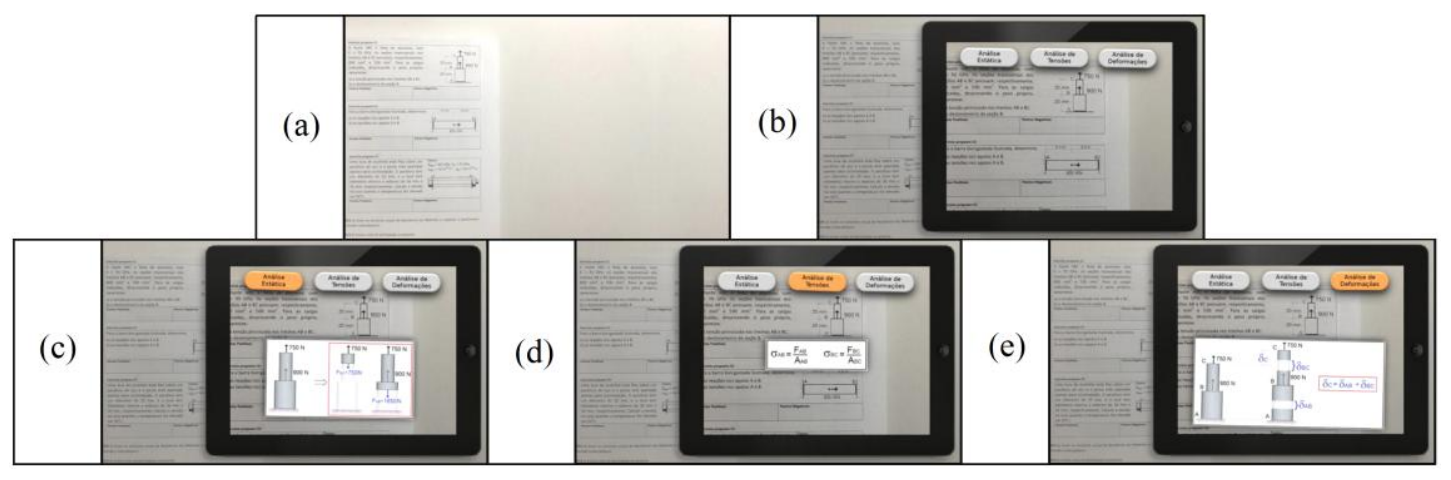

Figure 3. Imagens de um dos recursos desenvolvidos: (a) Roteiro com os três exemplos utilizados, (b) Menu apresentado em RA, (c) Análise Estática do exemplo focalizado, (d) Análise de Tensões, (e) Análise de Deformações. 
Durante o experimento, os participantes foram observados por um dos autores deste artigo e utilizaram dois tablets, disponibilizados para a atividade com a solução Aurasma (software adotado para o desenvolvimento dos recursos de RA [Souza e Pacheco, 2014], [Tori, 2014]).

Quadro 2. Declarações que constituíram o questionário.

\begin{tabular}{|c|c|}
\hline Construto & Questões \\
\hline \multirow{4}{*}{ Facilidade de Uso } & Q1: Usar os recursos de Realidade Aumentada - RA é fácil. \\
\hline & Q2: Aprender a usar os recursos de RA é fácil. \\
\hline & Q3: Não é necessário treinamento para utilizar os recursos de RA. \\
\hline & Q4: Estou acostumado(a) a utilizar os recursos de RA. \\
\hline \multirow{4}{*}{ Utilidade Percebida } & Q5: Os recursos de RA são úteis para o estudo do conteúdo da disciplina. \\
\hline & Q6: O uso de recursos de RA reduz o tempo para a compreensão do conteúdo. \\
\hline & Q7: O uso de recursos de RA melhoram a qualidade da compreensão do conteúdo. \\
\hline & Q8: O uso de recursos de RA em outras disciplinas é importante para a minha formação. \\
\hline \multirow{4}{*}{$\begin{array}{l}\text { Intenção Comportamental } \\
\text { de Uso }\end{array}$} & Q9: Recomendo a utilização de recursos de RA na disciplina Resistência dos Materiais. \\
\hline & Q10: Gostaria que a disciplina Resistência dos Materiais adotasse os recursos de RA. \\
\hline & Q11: Gostaria que outras disciplinas do curso de Engenharia adotassem os recursos de RA. \\
\hline & Q12: Estou motivado(a) a utilizar os recursos de RA. \\
\hline \multirow{2}{*}{ Variável 01: Treinamento } & Q13: As orientações do instrutor/professor para a utilização dos recursos de RA foram adequadas. \\
\hline & Q14: O roteiro fornecido para o uso dos recursos de RA é fácil de ser compreendido. \\
\hline \multirow{2}{*}{$\begin{array}{l}\text { Variável 02: Características } \\
\text { da Interface }\end{array}$} & Q15: Os recursos de RA utilizados apresentaram visual agradável. \\
\hline & Q16: Os menus e as imagens dos recursos de RA utilizados eram claros e objetivos. \\
\hline \multirow{2}{*}{$\begin{array}{l}\text { Variável 03: Custo da } \\
\text { utilização }\end{array}$} & Q17: Tenho dispositivo móvel com sistema IOS ou Android, que permite acessar os recursos de RA. \\
\hline & Q18: Tenho acesso à internet e o acesso aos recursos de RA não representa custo adicional. \\
\hline
\end{tabular}

O questionário foi submetido aos estudantes por meio eletrônico, no ambiente virtual de aprendizagem da disciplina. O recurso utilizado para construção deste instrumento de pesquisa foi o módulo feedback, um dos diversos disponíveis no Moodle $^{1}$ (plataforma de utilizada para construção do ambiente virtual da disciplina). O referido módulo permite a elaboração de formulários online e dispõe de recursos para a tabulação dos resultados, o que facilitou a coleta e análise dos dados.

A estrutura do questionário foi composta por uma questão para identificar o curso do discente e dezoito declarações, associadas a escalas de Likert, (1 - Discordo Completamente, 2 - Discordo, 3 - Não Sei/Indiferente, 4 - Concordo, 5 - Concordo Completamente) organizadas em blocos vinculados aos construtos do TAM (Quadro 2).

\section{Resultados}

O gráfico da Figura 4 apresenta indícios descritivos das respostas dos estudantes no questionário aplicado na pesquisa. A linha contínua representa a média das respostas fornecidas, enquanto a linha pontilhada representa o nível neutro da escala de Likert (Não Sei / Indiferente $=3$ ). Esse gráfico fornece um panorama geral da opinião dos estudantes após participarem do experimento que foi realizado e demonstra que a maioria absoluta das médias de respostas das declarações (Quadro 2) está acima do nível neutro.

Os dois itens de maior média foram Q9 e Q13 com, respectivamente, 4,80 e 4,68 (médias acima do nível 'concordo'), demonstrando que os estudantes tiveram uma percepção positiva em relação à utilidade de RA na disciplina e que as orientações fornecidas no experimento foram adequadas para a utilização dos recursos.

\footnotetext{
${ }^{1} \mathrm{http} / / /$ docs.moodle.org/23/en/Feedback_module
} 
CBIE-LACLO 2015

Anais dos Workshops do IV Congresso Brasileiro de Informática na Educação (CBIE 2015)

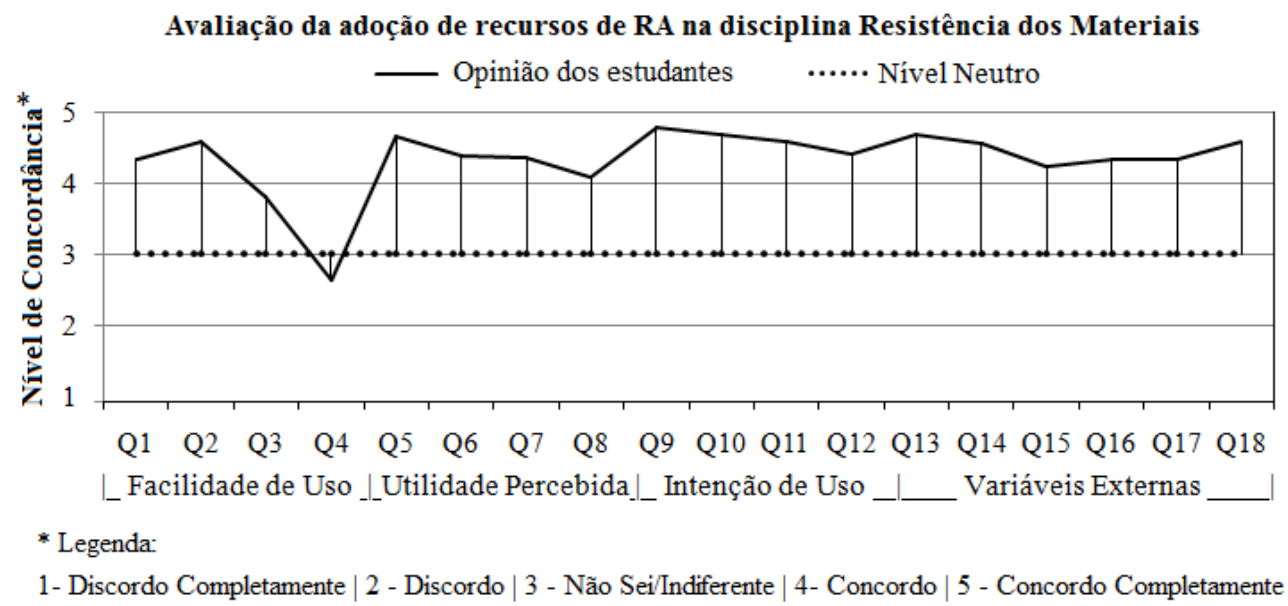

Figure 4. Gráfico com as médias das respostas do questionário, obtidas a partir da transformação dos dados categóricos da escalas de Likert em dados numéricos.

A única declaração que obteve uma média abaixo do nível neutro foi a $\mathrm{Q} 4$, com o enunciado "Estou acostumado(a) a utilizar os recursos de RA". De acordo com o gráfico, entre os estudantes que participaram da pesquisa, uma maior parcela declarou não estar habituada a utilizar esse tipo de recurso.

Embora as evidências da aceitação dos recursos de RA na disciplina sejam perceptíveis graficamente, para detalhar os dados coletados, a distribuição de frequência das respostas dos estudantes (Tabela 1) é discutida nas próximas seções.

Tabela 1. Distribuição de frequência das respostas.

\begin{tabular}{|c|c|c|c|c|c|c|c|}
\hline \multirow{2}{*}{\multicolumn{2}{|c|}{ Declaração }} & \multicolumn{6}{|c|}{ Nível de Concordância } \\
\hline & & 1 & 2 & 3 & 4 & 5 & Total \\
\hline \multirow{4}{*}{ Facilidade de Uso } & Q1 & $0 \%$ & $4 \%$ & $0 \%$ & $54 \%$ & $42 \%$ & $100 \%$ \\
\hline & Q2 & $0 \%$ & $0 \%$ & $0 \%$ & $42 \%$ & $58 \%$ & $100 \%$ \\
\hline & Q3 & $0 \%$ & $18 \%$ & $10 \%$ & $44 \%$ & $28 \%$ & $100 \%$ \\
\hline & Q4 & $18 \%$ & $36 \%$ & $12 \%$ & $32 \%$ & $2 \%$ & $100 \%$ \\
\hline \multirow{4}{*}{ Utilidade Percebida } & Q5 & $0 \%$ & $0 \%$ & $4 \%$ & $26 \%$ & $70 \%$ & $100 \%$ \\
\hline & Q6 & $0 \%$ & $2 \%$ & $8 \%$ & $40 \%$ & $50 \%$ & $100 \%$ \\
\hline & Q7 & $0 \%$ & $4 \%$ & $4 \%$ & $44 \%$ & $48 \%$ & $100 \%$ \\
\hline & Q8 & $0 \%$ & $8 \%$ & $12 \%$ & $44 \%$ & $36 \%$ & $100 \%$ \\
\hline \multirow{4}{*}{ Intenção de Uso } & Q9 & $0 \%$ & $0 \%$ & $0 \%$ & $20 \%$ & $80 \%$ & $100 \%$ \\
\hline & Q10 & $0 \%$ & $0 \%$ & $2 \%$ & $26 \%$ & $72 \%$ & $100 \%$ \\
\hline & Q11 & $0 \%$ & $0 \%$ & $2 \%$ & $36 \%$ & $62 \%$ & $100 \%$ \\
\hline & Q12 & $0 \%$ & $2 \%$ & $8 \%$ & $36 \%$ & $54 \%$ & $100 \%$ \\
\hline \multirow{6}{*}{ Variáveis Externas } & Q13 & $0 \%$ & $0 \%$ & $2 \%$ & $28 \%$ & $70 \%$ & $100 \%$ \\
\hline & Q14 & $0 \%$ & $0 \%$ & $0 \%$ & $44 \%$ & $56 \%$ & $100 \%$ \\
\hline & Q15 & $0 \%$ & $2 \%$ & $4 \%$ & $62 \%$ & $32 \%$ & $100 \%$ \\
\hline & Q16 & $0 \%$ & $2 \%$ & $4 \%$ & $52 \%$ & $42 \%$ & $100 \%$ \\
\hline & Q17 & $8 \%$ & $4 \%$ & $0 \%$ & $22 \%$ & $66 \%$ & $100 \%$ \\
\hline & Q18 & $2 \%$ & $0 \%$ & $4 \%$ & $26 \%$ & $68 \%$ & $100 \%$ \\
\hline
\end{tabular}

\subsection{Facilidade de Uso Percebida}

No construto facilidade de uso percebida, foi medido o quanto o estudante acredita que utilizar os recursos de RA é fácil e, portanto, livre de esforço.

Analisando individualmente as questões dessa categoria, observa-se que $96 \%$ dos respondentes manifestaram que é fácil usar os recursos de RA, 100\% afirmam que é fácil aprender a usar e, para $72 \%$, não é necessário treinamento. Destaca-se que esses números foram obtidos mesmo com $66 \%$ dos respondentes informando que não estão acostumados a utilizar RA. 
CBIE-LACLO 2015

Anais dos Workshops do IV Congresso Brasileiro de Informática na Educação (CBIE 2015)

\subsection{Utilidade Percebida}

Com o construto utilidade percebida foi verificado o quanto o discente confia que utilizar os recursos de RA irá melhorar seu desempenho em Resistência dos Materiais.

Constata-se que $96 \%$ dos estudantes acreditam que os recursos de RA são úteis para o estudo do conteúdo da disciplina, $90 \%$ estimam que o uso de RA pode reduzir o tempo para a compreensão do conteúdo e, para 92\%, a adoção desse tipo de material melhora a qualidade da compreensão do conteúdo. Além disso, 80\% dos estudantes percebem que RA é útil em outras disciplinas e importante para a formação acadêmica.

\subsection{Intenção de Uso}

A análise da intenção de uso foi realiza com o propósito de identificar se existe, por parte dos estudantes, interesse em utilizar os recursos de RA em Resistência dos Materiais e em outras disciplinas, possibilitando a identificação de mais indícios da aceitação ou não dessa tecnologia pelos discentes.

Nesse sentido, $100 \%$ dos participantes da pesquisa recomendaram o uso dos recursos em Resistência dos Materiais e 98\% declararam que gostariam que outras disciplinas do curso de Engenharia utilizassem RA.

\subsection{Variáveis Externas}

A análise das variáveis externas foi realizada com o objetivo de compreender melhor quais fatores influenciam na facilidade percebida e na utilidade percebida. A escolha dessas variáveis foi fundamentada em aspectos identificados na revisão da literatura do TAM. Nesta pesquisa, as variáveis externas utilizadas foram o treinamento, as características da interface dos recursos e o custo da utilização.

Mesmo com uma orientação muito breve, pautada em um roteiro de apenas uma página, 98\% dos estudantes afirmaram que as orientações foram adequadas e, para $100 \%$ dos discentes, o roteiro foi de fácil compreensão.

Em relação à interface dos recursos utilizados no experimento, $94 \%$ dos respondentes avaliaram que os menus e as imagens eram claros, objetivo e que, além disso, possuíam um visual agradável.

Nas declarações sobre aspectos relacionados aos custos para a utilização dos recursos, $12 \%$ dos estudantes disseram não possuir o dispositivo móvel necessário para acessar o conteúdo em RA e, por conseqüência, teriam que comprar. A respeito da internet, apesar de 6\% dos estudantes não confirmarem a disponibilidade de acesso a rede mundial de computadores, na Univasf os discentes podem obter esse acesso sem custos, a partir de cadastro junto à Secretaria de Tecnologia da Informação (STI/Univasf).

\section{Considerações finais e trabalhos futuros}

Neste estudo, foram analisados dados coletados por meio de um questionário com questões organizadas em blocos vinculados aos construtos do Modelo de Aceitação de Tecnologia - TAM, respondidas por 50 (cinquenta) estudantes da disciplina Resistência dos Materiais ofertada pela Universidade Federal do Vale do São Francisco, com o objetivo de investigar o potencial para adoção de recursos de RA no ensino de Engenharia. 


\section{CBIE-LACLO 2015}

Anais dos Workshops do IV Congresso Brasileiro de Informática na Educação (CBIE 2015)

Verificou-se que, na opinião da maioria absoluta dos estudantes, a RA é uma tecnologia de fácil utilização e útil para a construção do conhecimento em Resistência dos Materiais, potencializando o trabalho docente na exposição do conteúdo e, simultaneamente, ajudando os discentes na compreensão dos assuntos abordados na disciplina. Como uma manifestação expressiva da aceitação dessa tecnologia no âmbito pedagógico, $100 \%$ dos participantes da pesquisa recomendaram a adoção de RA entre os recursos apoio da componente curricular.

Apesar de apresentar resultados significativamente positivos, os procedimentos adotados na pesquisa permitiram apenas a coleta de dados relacionados a um experimento específico, não oferecendo elementos suficientes para avaliar detalhadamente a motivação dos estudantes em relação ao uso dos recursos de RA na disciplina Resistência dos Materiais. Essa limitação impede, por exemplo, afirmar quais são as causas que levaram alguns estudantes a discordarem que os recursos de RA melhoram a qualidade da compreensão do conteúdo. Nesse sentido, apesar de ter alcançado o objetivo estabelecido nesta pesquisa, vários outros trabalhos podem ser realizados para aprofundar os estudos sobre o uso de Realidade Aumentada no ensino de Engenharia.

Em trabalhos futuros, pretende-se desenvolver novos recursos de RA para Resistência dos Materiais a partir das diretrizes de Interfaces Naturais de Usuário e, em ambiente de sala de aula, realizar experimentos para avaliar os principais fatores que afetam a percepção dos estudantes. Além disso, serão estudadas metodologias para estimular que os estudantes também produzam recursos que possam ser compartilhados e utilizados na disciplina.

\section{Referências}

Ayeh, J; Au, N.; Law, R. (2013). Predicting the intention to use consumer-generated media for travel planning. Tourism Management 35: 132-143.

Blake, J. (2013). Natural User Interfaces. Manning Pubs Series, Manning Publications Company.

Dantas, A. D.; Maciel, L.; Lima, D., Castro, T.; Lima, I.; Neto, A. R. (2014). Aplicando o Sloodle como um Ambiente de Ensino e Aprendizagem. In Anais do Workshop de Ambientes Virtuais na Educação. Congresso Brasileiro de Informática na Educação.

Davis, F. (1989). Perceived usefulness, perceived ease of use, and user acceptance of information technology. MIS quarterly. 319-340.

Dias, G. (2011). Technology Acceptance Model (TAM): avaliando a aceitação tecnológica do Open Journal Systems (OJS). Informação \& Sociedade: Estudos 21.2.

Haguenauer, C. (2012). Ambiente colaborativo na internet. Disponível em: http://www.latec.ufrj.br/at.htm\#ambiente. Acesso em 10 de abril de 2015.

Haugstvedt, A., Krogstie, J. (2012). Mobile augmented reality for cultural heritage: A technology acceptance study. In: ISMAR, 2012 IEEE International Symposium.

Kawamoto, A. (2013). Utilização de Dispositivos de Interfaces Naturais de Usuário em Aplicações de realidade aumentada. In: Tendências e técnicas em realidade virtual e aumentada, v. 3, p. 75-88. 


\section{CBIE-LACLO 2015}

Anais dos Workshops do IV Congresso Brasileiro de Informática na Educação (CBIE 2015)

Kirner, C.; Kirner, T. G. (2007) Virtual Reality and Augmented Reality Applied to Simulation Visualization. In: El Sheikh, A.A.R.; Al Ajeeli, A.; Abu-Taieh, E.M.O.. (Org.). Simulation and Modeling: Current Technologies and Applications. 1 ed. Hershey-NY: IGI Publishing, v. 1, p. 391-419.

Kruijff, E.; Swan I.; Feiner, S. (2010). Perceptual issues in augmented reality revisited. In: ISMAR, 2010 IEEE International Symposium.

Milgram , P.; Takemura , H.; Utsum i, A.; Kishino, F. (1994) Augmented Reality: A Class of Displays on the Reality-Virtuality Continuum, Telemanipulator and Telepresence Technologies, SPIE, V.2351.

Normand, J; Moreau, G. (2012). DoF-based Classification of Augmented Reality Applications. In. ISMAR, 2012 IEEE International Symposium.

Persico, D; Manca, S; Pozzi, F. (2014). Adapting the Technology Acceptance Model to evaluate the innovative potential of e-learning systems. Computers in Human Behavior 30: 614-622.

Santos, J. (1983). Sobre a concepção, o projeto, a execução e a utilização de modelos físicos qualitativos na engenharia de estruturas. Diss. Tese de Mestrado, USP.

Silva, J; Gomes, A. (2013). Estratégias para Blended Learning na Disciplina Resistência dos Materiais. XLI Congresso Brasileiro de Ensino em Engenharia. Gramado.

Souza, I.; Pacheco, B. (2014). O Uso de Dispositivos Móveis e Realidade Aumentada em Ambientes Virtuais de Ensino e Aprendizagem. In: Tendências e Técnicas em Realidade Virtual e Aumentada. Salvador: Sociedade Brasileira de Computação SBC. v. 4, p. 152-166.

Tori, R. (2014) Games e Realidade Aumentada para uma Educação sem Distância. Disponível em: <http://romerotori.blogspot.com.br/2014_05_01_archive.html>. Acesso em: 15 jun. 2015.

Valente, J. (2005). Pesquisa, comunicação e aprendizagem com o computador. In: Integração das Tecnologias na Educação. Secretaria de Educação a Distância. Brasília: Ministério da Educação, Seed, p. 22-30.

Wojciechowski, R; Cellary, W. (2013). Evaluation of learners' attitude toward learning in ARIES augmented reality environments. Computers \& Education 68: 570-585. 